\title{
Mayor presión y área de contacto en reparación transósea cruzada de Manguito Rotador versus reparación simple
}

\section{Increased Pressure and Contact Area in Rotator Cuff Crossed Transosseous Repair versus Simple Repair}

\author{
Julio José Contreras ${ }^{1}$ Rodrigo Liendo ${ }^{2}$ Francisco Soza ${ }^{2}$ \\ ${ }^{1}$ Instituto Traumatológico, Santiago, RM, Chile \\ 2 Pontificia Universidad Católica de Chile, Santiago, RM, Chile \\ Dirección para correspondencia Julio José Contreras Fernández, \\ Instituto Traumatológico, Santiago, RM, Chile \\ (e-mail: Juliocontrerasmd@gmail.com).
} Rev Chil Ortop Traumatol 2021;62(3):e159-e167.

\begin{abstract}
Resumen
Palabras Clave

- manguito de los rotadores

- presión

- sutura

- técnicas de sutura

- traumatismos de los tendones

- tendones

Objetivo Comparar la presión y el área de contacto en la interfase tendón-huella de una reparación realizada con suturas transóseas simples y cruzadas.

Métodos Se utilizaron doce hombros de cordero para simular una rotura de manguito rotador. Se midió el área de contacto en la interfase tendón-huella con láminas sensibles a presión; luego, se midió la presión con un sensor digital. Se registró la presión basal durante la aplicación de carga cíclica y al final de la intervención. Se compararon 2 reparaciones: 2 túneles transóseos con nudos simples (TOS; $n=6$ ) y 2 túneles transóseos con nudos cruzados (TOC; $n=6$ ), utilizando FiberWire \#2. Se realizaron 1.400 ciclos, con una frecuencia $2,5 \mathrm{~Hz}$ y una carga de $5 \mathrm{~N}$. Se utilizó la prueba de Mann-Whitney, y ae consideraron significativos valores de $p<0,05$.

Resultados La reparación TOS presentó un 50,9 $\pm 12,7 \%$ de distribución de presiones en comparación con $72,2 \pm 5,3 \%$ en la reparación TOC $(p<0,009)$. La presión promedio en la reparación TOS fue $0,7 \pm 0,1 \mathrm{MPa}$ en comparación con 1,1 $\pm 0,2 \mathrm{MPa}$ en la reparación TOC $(p<0,007)$. La reparación TOS registró una presión basal de 5,3 $\pm 5,3$ $\mathrm{N}$, presión final de $3,8 \pm 4,6 \mathrm{~N}$, y una variación de $51,7 \pm 38 \%$. La reparación TOC registró una presión basal de $10,7 \pm 1,8 \mathrm{~N}$, presión final de $12,9 \pm 8,7 \mathrm{~N}$, y una variación de $114,9 \pm 65,9 \%$ ( $p<0,044 ; p<0,022$; y $p<0,017$, respectivamente).

Conclusión La reparación TOC presenta mayor presión a nivel de la interfase tendónhueso, menor pérdida de fuerza de contacto ante cargas cíclicas, y una mejor distribución de fuerza en la huella al comparar con la reparación TOS, lo que se podría traducir en mejor cicatrización tendínea.

Nivel de Evidencia Estudio de ciencia básica.
\end{abstract}

recibido

28 de junio de 2020

aceptado

06 de agosto de 2021
DOI https://doi.org/

10.1055/s-0041-1740546. ISSN 0716-4548.
(C) 2021. Sociedad Chilena de Ortopedia y Traumatologia. All rights reserved.

This is an open access article published by Thieme under the terms of the Creative Commons Attribution-NonDerivative-NonCommercial-License, permitting copying and reproduction so long as the original work is given appropriate credit. Contents may not be used for commercial purposes, or adapted, remixed, transformed or built upon. (https://creativecommons.org/ licenses/by-nc-nd/4.0/)

Thieme Revinter Publicações Ltda., Rua do Matoso 170, Rio de Janeiro, RJ, CEP 20270-135, Brazil 


\begin{abstract}
Keywords

- rotator cuff

- pressure

- suture

- suture techniques

- tendon injuries

- tendons

Objective To compare the pressure and contact area at the tendon-footprint interface of a repair performed with simple and crossed transosseous sutures.

Methods Twelve lamb shoulders were used to simulate a rotator cuff tear. The contact area at the tendon-footprint interface was measured with pressure-sensitive films; then, the pressure was measured with a digital sensor. The baseline pressure was recorded during the application of a cyclic load and at the end of the intervention. A total of 2 repairs were compared: 2 transosseous sutures with single knots (TOS; $n=6$ ) and 2 transosseous sutures with crossed knots (TOC; $n=6$ ) using FiberWire \#2. In total, 1,400 cycles were performed, with a frequency of $2.5 \mathrm{~Hz}$ and a load of $5 \mathrm{~N}$. The MannWhitney test was used. Values of $p<0.05$ were considered significant.

Results The TOS repair presented $50.9 \pm 12.7 \%$ of pressure distribution compared to $72.2 \pm 5.3 \%$ in the TOC repair $(p<0.009)$. The mean pressure in the STO repair was of $0.7 \pm 0.1 \mathrm{MPa}$ compared to $1.1 \pm 0.2 \mathrm{MPa}$ in the TOC repair $(p<0.007)$. The STO repair registered a basal pressure of $5.3 \pm 5.3 \mathrm{~N}$, a final pressure of $3.8 \pm 4.6 \mathrm{~N}$, and a variation of $51.7 \pm 38 \%$. The TOC repair registered a basal pressure of $10.7 \pm 1.8 \mathrm{~N}$, a final pressure of $12.9 \pm 8.7 \mathrm{~N}$, and a variation of $114.9 \pm 65.9 \%(p<0.044 ; p<0.022$; and $p<0.017$ respectively).

Conclusion The TOC repair presents higher pressure at the tendon-bone interface, less loss of contact force under cyclic loads, and a better distribution of force on the footprint when compared with the TOS repair, which could translate into better tendon healing.

Level of Evidence Basic Science Study.
\end{abstract}

\section{Introducción}

La reparación artroscópica del manguito rotador ha presentado un aumento constante en el último tiempo. ${ }^{1}$ Los resultados clínicos y funcionales son de buenos a excelentes en el corto y largo plazos en la mayoría de los casos: ${ }^{2-5}$ sin embargo, las tasas de rerrotura siguen siendo considerables, descritas entre un $11 \%$ y un $68 \%$ en algunas series, incluso alcanzando un $94 \%$ en algunos estudios. ${ }^{6-8}$

La cirugía de reparación del manguito rotador busca establecer una interfase fibrovascular entre el tendón y la huella, que es necesaria para la curación y la restauración de la inserción fibrocartilaginosa (entesis); para lograr esto, se busca un constructo que maximice el contacto presurizado entre el tendón y el hueso mientras mantiene la resistencia mecánica contra la carga fisiológica. ${ }^{9}, 10$

Respecto a los factores anatómicos que favorecen la cicatrización, podemos mencionar una adecuada tensión del constructo y perfusión tisular, un micromovimiento reducido en la interfase tendón-huella, y las adecuadas presión y área de contacto en la huella. ${ }^{11}$ El principio subyacente es el de que una mayor magnitud y distribución del área de contacto del tendón al hueso dará como resultado una mayor posibilidad de curación del tendón. $^{12}$

La reparación en doble fila (DF) presenta un aumento de la resistencia a la falla por carga, áreas de contacto y presiones mejoradas, y una disminución de la formación de espacios (gaps) en la interfase tendón-huella en comparación con la reparación en fila única $(\mathrm{FU}){ }^{5,13}$ Sin embargo, las anclas proporcionan una baja resistencia, tienden a aflojarse en el hueso osteoporótico, dejan de tener un contacto óptimo a nivel de la huella del tendón supraespinoso, producen osteólisis de la tuberosidad mayor, son de revisión difícil, y generan aumento del costo. ${ }^{14-18}$

La técnica transósea (TO) permite maximizar el área de contacto de la interfase tendón-huella ${ }^{19}$ y reducir el movimiento en la interfase tendón-hueso. ${ }^{20}$ Además de este aspecto mecánico, la técnica TO permite preparar con precisión el lado óseo de la lesión sin ningún riesgo o complicación, como la extracción del ancla y/o una osteólisis de la tuberosidad mayor. ${ }^{21,22}$

El uso de técnicas de sutura TO es eficiente y reproducible en la reparación artroscópica de roturas del manguito rotador. ${ }^{23}$ Además, existe un potencial mayor de cicatrización por el contacto directo del tendón con el hueso (sin material de anclas interpuesto) y con células madre mesenquimales provenientes de los túneles óseos del húmero proximal. ${ }^{24-27}$

En relación con estas ventajas, nuestro equipo diseñó un dispositivo capaz de realizar túneles TOs de arquitectura oblicua, con opción de realizar reparación con suturas simples o cruzadas.

El objetivo de este estudio es comparar la presión y el área de contacto en la interfase tendón-huella de una reparación realizada con suturas TOs simples y cruzadas. Nuestra hipótesis es que la configuración cruzada tendrá una mayor área de contacto y una menor disminución de presión tras carga cíclica. 


\section{Materiales y Métodos}

\section{Modelo Animal}

Um total de 12 hombros de cordero frescos congelados (Ovis orientalis aries) de 6 meses de edad, obtenidos de una empresa frigorífica local (corte paleta oyster, Frigorífico Simunovic Ltda., Punta Arenas, Región de Magallanes y Antártica Chilena, Chile), fueron descongelados a temperatura ambiente la noche previa a las pruebas biomecánicas. Se seleccionó el tendón infraespinoso por poseer características anatómicas y funcionales equivalentes a las del tendón supraespinoso de humanos. ${ }^{28}$ Los especímenes fueron disecados de forma estandarizada, eliminando todo el tejido blando adyacente a la diáfisis humeral, las fosas subescapular y supraespinosa de la escápula, con el objetivo de aislar el músculo infraespinoso y su tendón. No se evidenciaron alteraciones del manguito rotador en ninguno de los especímenes. Luego, se realizó una osteotomía de la escápula a nivel de la espina, conservando la inserción del músculo infraespinoso, con tal de poder manipular el músculo sin desgarrarlo (-Figura 1). Finalmente, se realizaron dos perforaciones a $1 \mathrm{~cm}$ del borde medial de la escápula y separadas por $1 \mathrm{~cm}$ a cada lado de la espina escapular con una broca $5.0 \mathrm{~mm}$, para poder enganchar el fragmento osteotomizado a la celda de carga ( - Figura 2 y 3 ). Las piezas fueron irrigadas intermitentemente con solución de $\mathrm{NaCl}$ 0,9\% a lo largo de cada ensayo para prevenir la deshidratación de la muestra.

Se utilizó un sistema a medida para generar tensiones cíclicas a nivel de músculo y tendón

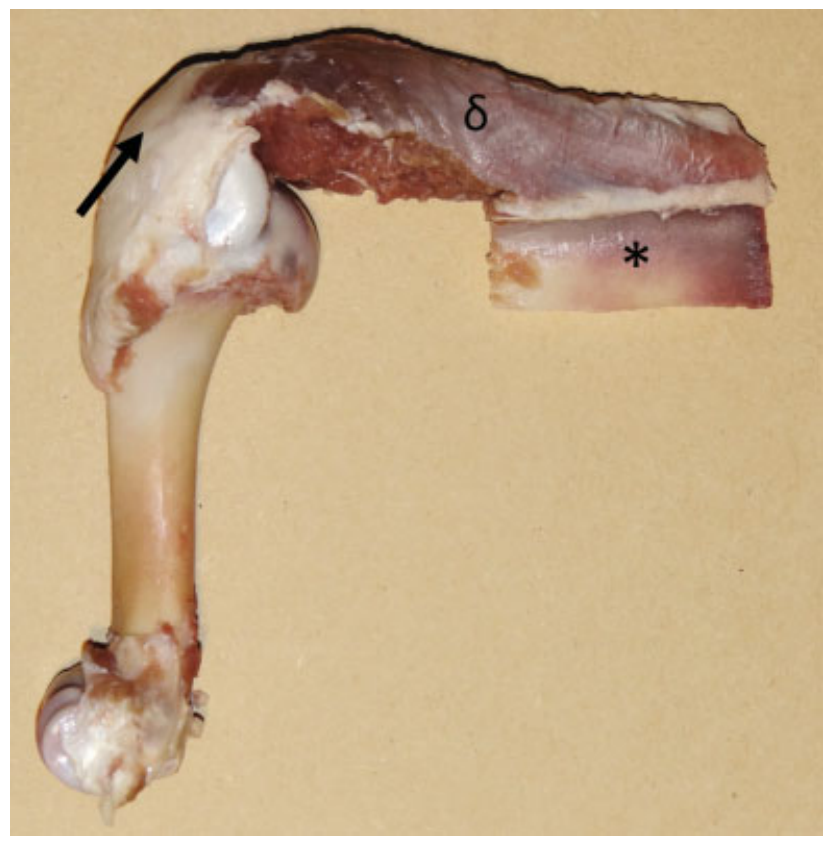

Fig. 1 Disección anatómica de tendón infraespinoso de cordero. Disección anatómica estandarizada, eliminando todo el tejido blando adyacente a la diáfisis humeral, y las fosas subescapular y supraespinosa de la escápula, con el objetivo de aislar el músculo infraespinoso y su tendón. En la figura, la flecha indica el tendón infraespinoso, el delta $(\delta)$, el músculo infraespinoso, y el asterisco, la osteotomía de la escápula a nivel de la espina, conservando la inserción del músculo infraespinoso.

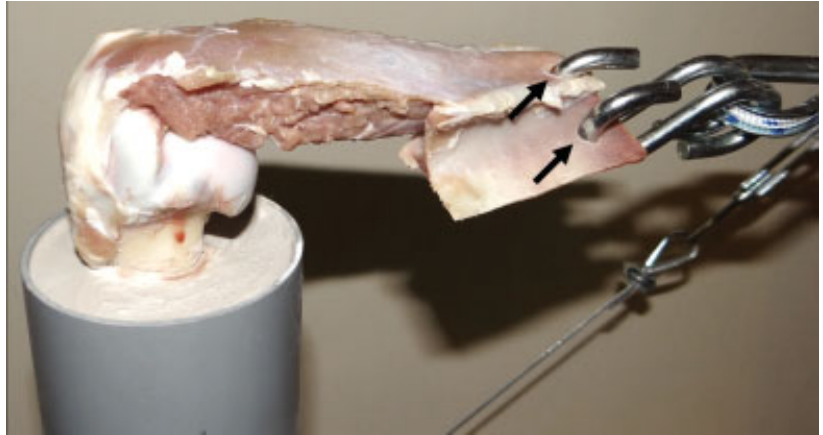

Fig. 2 Agujeros escapulares para fijación del músculo infraespinoso (vista anterior). Se realizan 2 perforaciones a $1 \mathrm{~cm}$ del borde medial de la escápula y separadas por $1 \mathrm{~cm}$ a cada lado de la espina escapular con una broca $5.0 \mathrm{~mm}$, para poder enganchar el fragmento osteotomizado a la celda de carga (visión anterior).

infraespinoso (-Figura 4). El modelo constó de tres partes fundamentales: un soporte modular de altura regulable, un soporte ajustable para guiar el sistema de suturas, y una celda de carga regulada digitalmente por el programa Arduino (código abierto).

La diáfisis humeral fue fijada en un cilindro plástico de policloruro de vinilo (polyvinyl chloride, PVC, en inglés) con yeso. Luego, se ajustó el soporte modular para asegurar el

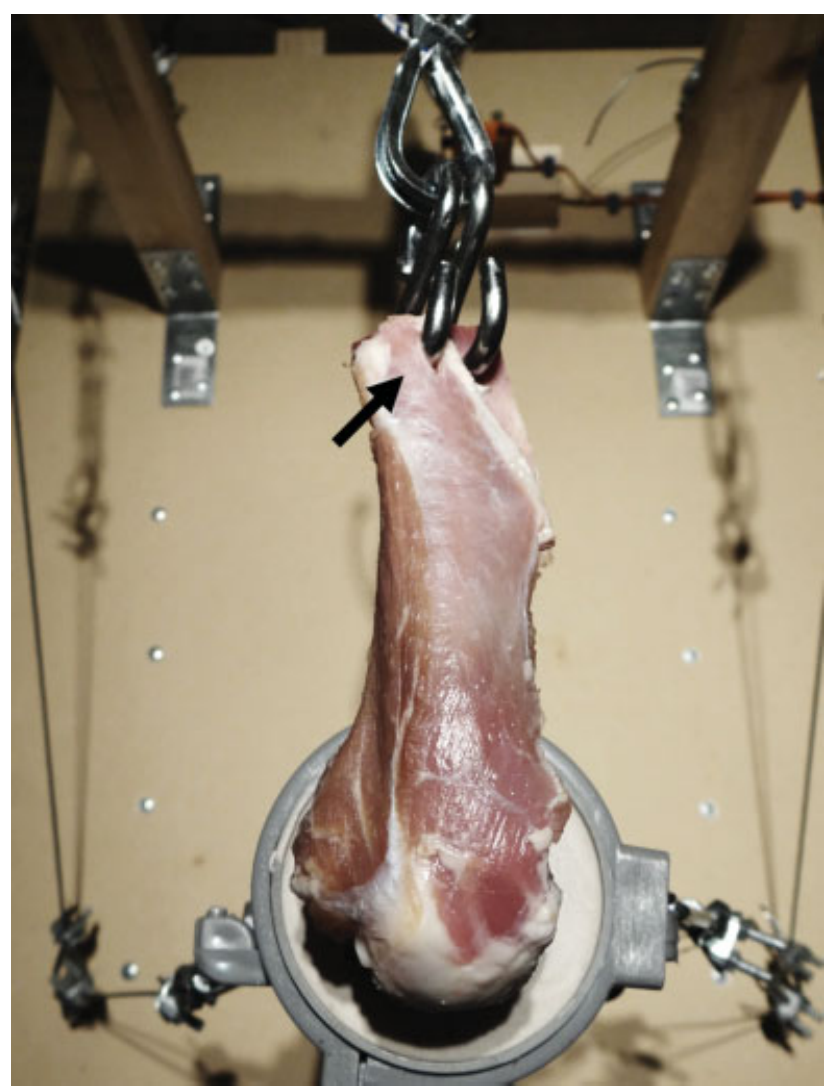

Fig. 3 Agujeros escapulares para fijación del músculo infraespinoso (vista superior). Se realizan 2 perforaciones a $1 \mathrm{~cm}$ del borde medial de la escápula y separadas por $1 \mathrm{~cm}$ a cada lado de la espina escapular con una broca $5.0 \mathrm{~mm}$, para poder enganchar el fragmento osteotomizado a la celda de carga (visión superior). La flecha muestra como el músculo se mantiene indemne, sin desgarros. 


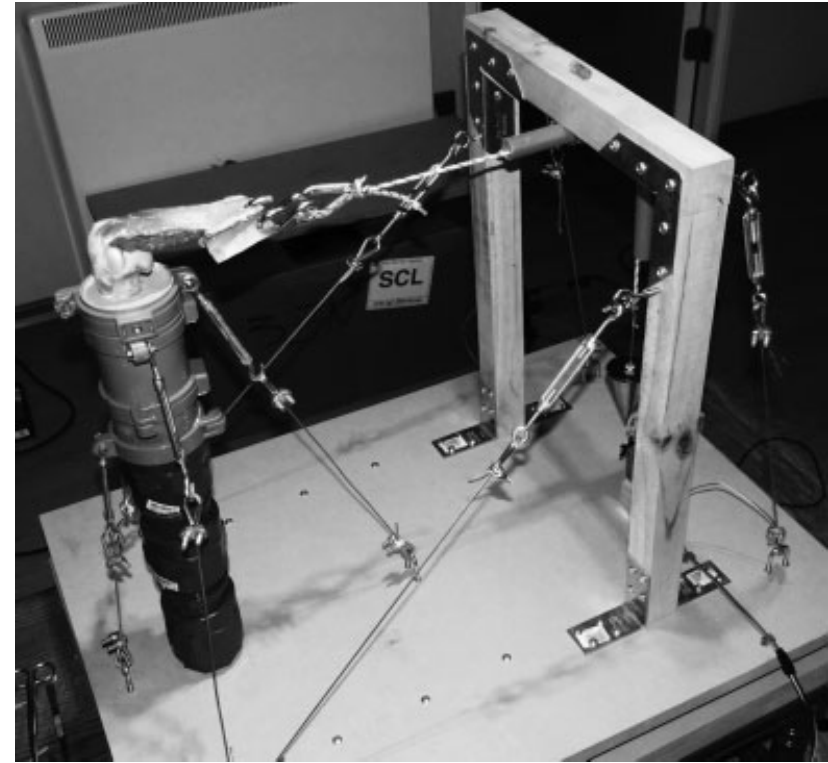

Fig. 4 Modelo de tensión cíclica. El modelo constó de un soporte modular de altura regulable, un soporte ajustable para guiar el sistema de suturas, y una celda de carga. La diáfisis humeral fue fijada en un cilindro plástico de PVC con yeso.

paralelismo del tendón con la horizontal (se utilizó un nivel), y se logró un ángulo de tracción de $0^{\circ}$ de abducción.

\section{Rotura del Manguito Rotador}

En cada cabeza humeral, se identificó la orientación de la tuberosidad mayor y se demarcó con una aguja de Kirschner de $1,5 \mathrm{~mm}$. Seguidamente, se identificó la punta de la tuberosidad y, a $10 \mathrm{~mm}$ lateral, se realizó una rotura de espesor total y de $20 \mathrm{~mm}$ de ancho con un bisturí $\mathrm{N}^{\circ} 15$, liberando toda la inserción del tendón en la huella, para luego aplanarla con una raspa, con tal de facilitar la instalación de los sensores de presión (- Figura 5).

\section{Medición de Presión y Área de Contacto en la Interfase Tendón-huella}

Se midió el área de contacto al inicio de la reparación, y la presión, al inicio, durante, y al final de la aplicación de las cargas cíclicas.

Primero, en tiempo cero, se midió el área de contacto en la interfase tendón-huella (porcentaje y presión promedio, MPa). Para esto, se utilizó un conjunto de láminas sensibles a la presión con un registro colorimétrico (Prescale Ultra Low Pressure Fuji Photo Film, C. Itoh \& Co, Nueva York, NY, EEUU) recubiertas con un film plástico para protegerlas de la humedad de los tejidos; fueron posicionadas en la superficie previamente aplanada de la huella. Posteriormente, se procedió a la reparación. Luego, las láminas fueron digitalizadas y analizadas con un escáner y programa previamente calibrados (Fujifilm Analysis System for Prescale, Tekscan, Inc., South Boston, MA, EEUU).

Para medir la presión en la interfase tendón-huella, se utilizó un sensor de presión digital (Flexiforce Sensor, Tekscan), el cual se posicionó entre el tendón y la huella, y quedó fijo gracias a la reparación realizada; este sensor

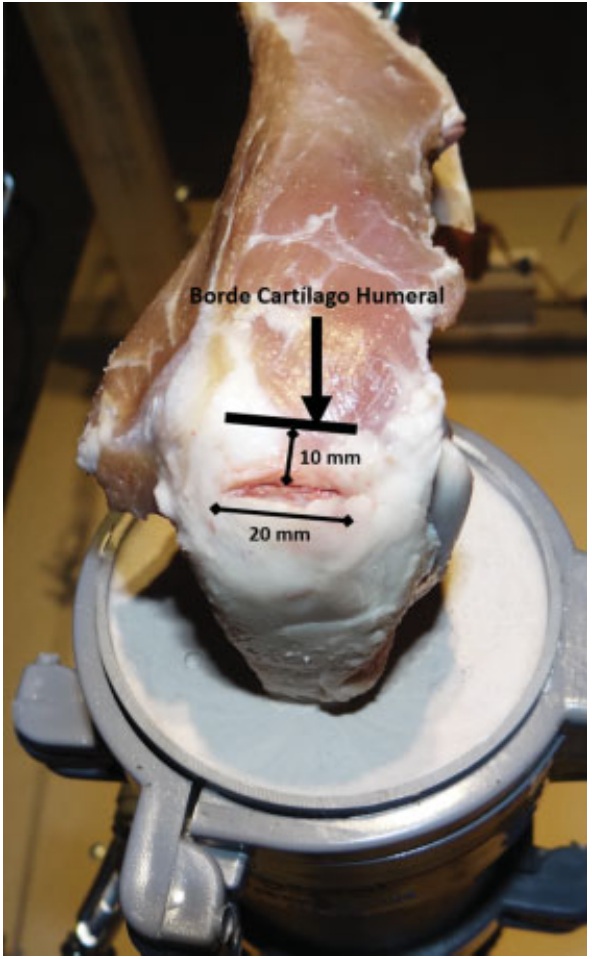

Fig. 5 Rotura del manguito rotador. La flecha demarca el borde del cartílago humeral, que se palpa y marca con una aguja. Luego, $10 \mathrm{~mm}$ lateral, se realiza una rotura de espesor total y de $20 \mathrm{~mm}$ de ancho.

registra los cambios de presión en el tiempo, y los almacena en un computador para su análisis posterior (unidad de medición, Newton $[\mathrm{N}]$ ). Se registró la presión basal al inicio del experimento (tiempo cero), durante la aplicación de carga cíclica, y al final de la intervención.

\section{Reparación de Rotura de Manguito Rotador con Suturas Transóseas y Prueba Biomecánica}

Para la reparación, se utilizó una sutura de polímero no absorbible trenzada de poliéster, con un núcleo de polietileno de cadena larga \#2 (FiberWire, Arthrex, Naples, FL, EEUU), correspondiente al tamaño más comúnmente utilizado en la cirugía artroscópica de hombro.

Los túneles TOs fueron realizados con un dispositivo previamente diseñado por nuestro equipo, y utilizado en modelos previos para la generación de túneles de arquitectura oblicua (-Figura 6).

Se realizaron dos reparaciones TOs diferentes, siempre por el mismo cirujano (JC), con tal de disminuir la variabilidad interoperador: 1) dos túneles TOs con nudos simples (TOS) ( - Figura 7A) en seis hombros de cordero; y 2) dos túneles TOs con nudos cruzados (TOC) (-Figura 7B) en los seis hombros restantes. En ambas reparaciones, se utilizó FiberWire \#2, nudos tipo Tennessee slider para suturas transóseas y Revo-SCOI para suturas cruzadas. Para el anudado, no se utilizó tensiómetro.

Se realizó una pretensionado de la reparación con $10 \mathrm{~N}$ por 2 minutos. Luego, se programó la celda de carga a 1.400 ciclos, con una frecuencia $2,5 \mathrm{~Hz}$ y una carga de $5 \mathrm{~N}$. Estos parámetros se definieron con base en los utilizados en 


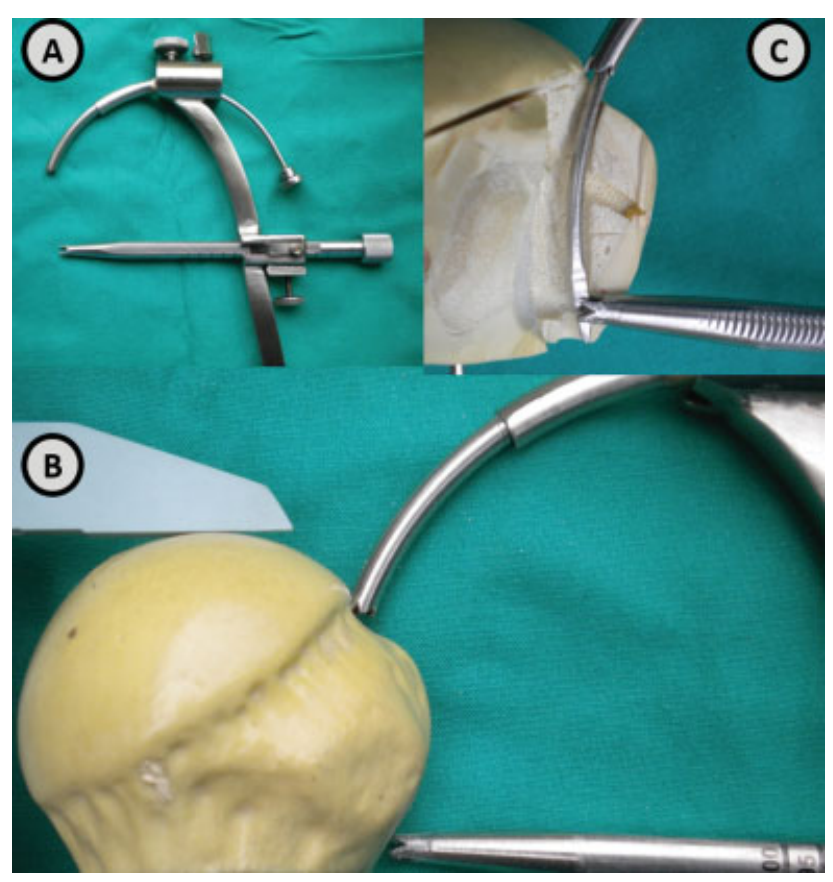

Fig. 6 Dispositivo para diseño de túneles transóseos oblicuos. En el recuadro $A$, se observa el dispositivo de suturas transóseas utilizado. En el recuadro $B$, se observa el posicionamiento adecuado del instrumento en relación con la tuberosidad mayor. Finalmente, en el recuadro $\mathrm{C}$, se observa un corte de un modelo óseo artificial que muestra la trayectoria oblicua del túnel.

estudios anteriores similares, ${ }^{29,30}$ y refleja el período de rehabilitación inicial (dos semanas) en el postoperatorio por medio de ejercicios pasivos con movimientos pendulares.
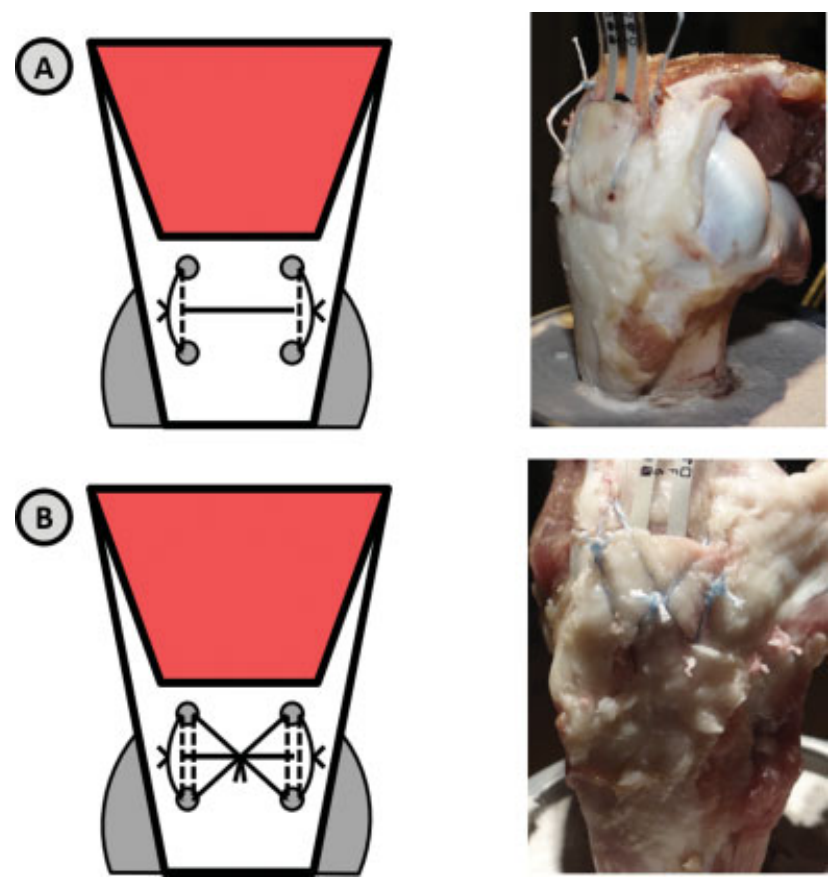

Fig. 7 Reparación con suturas transóseas. En el recuadro A, se observa la reparación transósea con nudos simples, y, en el B, la reparación con nudos cruzados.

\section{Análisis Estadístico}

Los resultados fueron presentados como promedios $\mathrm{y}$ desviaciones estándar. Dada una muestra pequeña, sin una distribución normal (la prueba de Shapiro-Wilk demostró una distribución no normal), se utilizó la prueba estadística para variables no paramétricas (test de Mann-Whitney). Todos los datos se analizaron utilizando el programa STATA (StataCorp LLC, College Station, TX, EEUU), versión 14 . Valores de $p<0,05$ fueron considerados estadísticamente significativos.

\section{Resultados}

Respecto al área de contacto en la interfase tendón-huella a tiempo cero, se observó una mayor área de distribución en el modelo de reparación TOC de 1,4 veces. La reparación TOS presentó un $50,9 \pm 12,7 \%$ de distribución de presiones en comparación con 72,2 $\pm 5,3 \%$ en la TOC ( $p<0,009)$ ( - Figura 8).

Respecto a la presión en la interfase tendón-huella a tiempo cero (medida con láminas sensibles a la presión), se observó una mayor presión de contacto en el modelo TOC de 1,6 veces. La PP en reparación TOS fue de 0,68 $\pm 0,13 \mathrm{MPa}$ en comparación con $1,1 \pm 0,2 \mathrm{MPa}$ en la reparación TOC $(p<0,007)$ (- Figura 8).

Respecto a la presión en la interfase tendón-huella en respuesta a carga cíclica (medida con sensor de presión digital), ambos modelos de reparación presentaron el mecanismo de autorreforzamiento (self-reinforcement) durante los aumentos de tensión cíclica por parte del sistema (-Figura 9).

La reparación TOS registró una presión basal de 5,3 $\pm 5,3 \mathrm{~N}$, una presión final de $3,8 \pm 4,6 \mathrm{~N}$, y una variación de $51,7 \pm 38 \%$ luego de 1.400 ciclos de tensión. La reparación TOC registró una presión basal de $10,7 \pm 1,8 \mathrm{~N}$, una presión final de $12,9 \pm 8,7 \mathrm{~N}$, y una variación de $114,9 \pm 65,9 \%(p<0,044$; $p<0,022$; y $p<0,017$, respectivamente) ( - Tabla 1 ).

\section{Discusión}

El hallazgo principal de nuestro estudio fue que la reparación TOC presenta mayor presión a nivel de la interfase tendón-hueso en tiempo cero, menor pérdida de fuerza de contacto ante cargas cíclicas en el tiempo, y una mejor distribución de fuerza en la huella al comparar con la reparación TOS.

Este hallazgo es un aporte importante, pues, en la utilización de instrumental artroscópico para la realización de suturas transóseas, el hecho de realizar la técnica con suturas cruzadas permitiría mejorar la presión y la distribución de fuerzas sólo modificando un gesto quirúrgico, siendo el primer reporte de esta ventaja biomecánica.

Los resultados obtenidos eran esperables una vez que la técnica de reparación TOC distribuyó la presión en una zona que no presentaba carga previamente; esto se relaciona directamente con el mecanismo de autorreforzamiento descrito por Burkhart et al. ${ }^{27}$ el año 2009, por el cual el aumento de la tensión aplicado al constructo genera una resistencia creciente a la falla estructural al generar un 

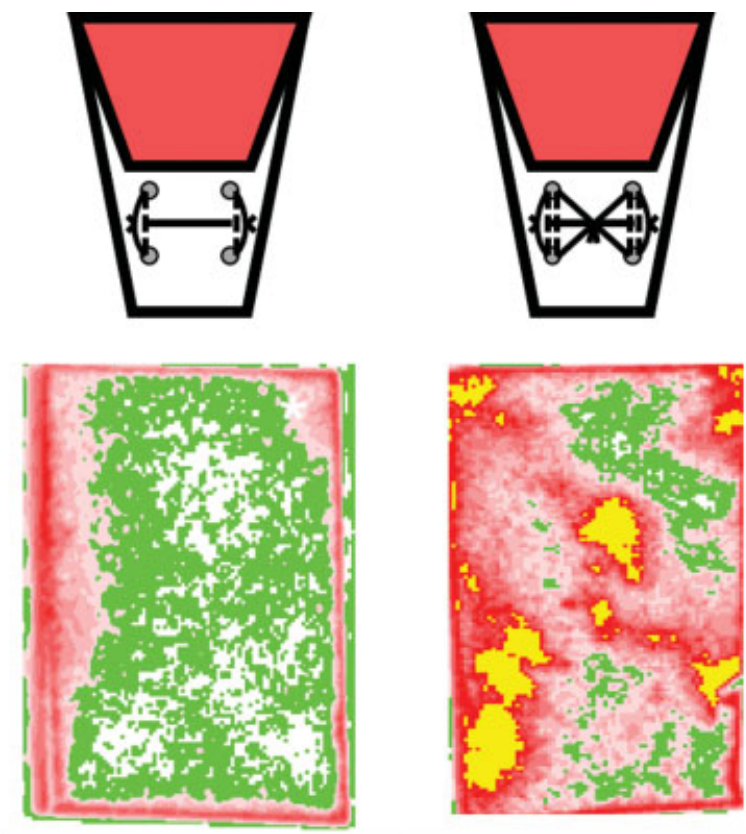

\begin{tabular}{|c|c|c|c|}
\hline Parámetro & Transósea simple & Transósea Cruzada & valor de $\boldsymbol{p}$ \\
\hline $\begin{array}{c}\text { Área de distribución de } \\
\text { presión }\end{array}$ & $50,94 \% \pm 12,69 \%$ & $72,24 \% \pm 5,32 \%$ & $\mathrm{p}<0,009$ \\
\hline Presión promedio & $0,68 \pm 0,1 \mathrm{~Pa}$ & $1,07 \pm 0,1 \mathrm{~Pa}$ & $\mathrm{p}<007$ \\
\hline Presión máxima & $2,74 \pm 0,45 \mathrm{~Pa}$ & $3,06 \pm 0,00 \mathrm{~Pa}$ \\
\hline
\end{tabular}

Fig. 8 Área de contacto y presión en la interfase tendón-huella a tiempo cero. Esta figura muestra el área de contacto medida con Fujifilm. El color rojo representa mayor presión de contacto, y el verde, una menor presión. Además, se incluye una tabla con los resultados cuantitativos, expresados en promedios y desviaciones estándar. Abreviatura: NS, no significativo.

aumento progresivo de las fuerzas de compresión en la huella del tendón. Las fuerzas de compresión creadas en la huella aumentan la resistencia a la fricción entre el tendón y el hueso, reduciendo así la formación de espacios entre las dos superficies. ${ }^{27}$ Se produce un acuñamiento del ángulo entre el material de sutura y el hueso a medida que el tendón se tensiona progresivamente, y un cambio de geometría rectangular a trapezoidal de las suturas en el plano coronal a medida que aumenta la carga de tracción. ${ }^{27}$ Esto provoca una deformación elástica del tendón y crea una fuerza de compresión perpendicular a la superficie del hueso, que aumenta a medida que aumenta la carga de tracción ${ }^{27}$ (-Figura 10).

Respecto a la alta desviación estándar reportada respecto a las presiones basal y final en ambos tipos de reparaciones, probablemente esto puede estar asociado a la tensión de los

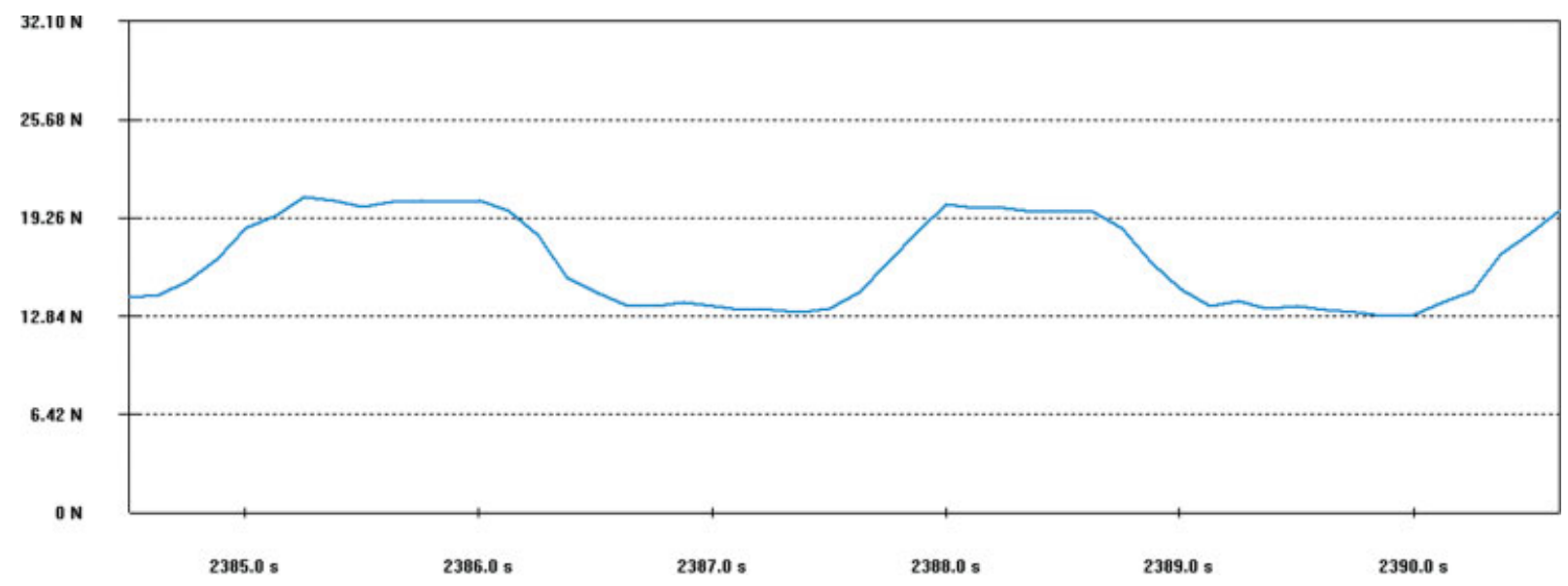

Fig. 9 Ejemplo de medición de la presión ante cargas cíclicas que demuestra el mecanismo de autorreforzamiento en reparación transósea. 
Tabla 1 Presión durante cargas cíclicas

\begin{tabular}{|l|l|l|l|l|}
\hline \multirow{2}{*}{} & \multicolumn{4}{|l|}{ Tipo de reparación } \\
\cline { 2 - 5 } & \multicolumn{2}{|l|}{ Transósea simple } & \multicolumn{2}{l|}{ Transósea Cruzada } \\
\hline Medición (N) & Promedio & DS & Promedio & DS \\
\hline Presión basal & 5,30 & 5,30 & 10,71 & 1,78 \\
\hline Presión 25\% & 4,91 & 5,59 & 10,88 & 4,95 \\
\hline Pico 25\% & 7,45 & 7,68 & 16,36 & 6,48 \\
\hline Presión 50\% & 5,18 & 6,63 & 13,12 & 8,09 \\
\hline Pico 50\% & 7,34 & 5,01 & 17,71 & 7,36 \\
\hline Presión 75\% & 4,63 & 6,54 & 11,98 & 6,19 \\
\hline Pico 75\% & 7,25 & 4,56 & 16,52 & 5,80 \\
\hline Presión Final & 3,84 & 1,34 & 12,90 & 8,73 \\
\hline Pico Final & 6,42 & $38,00 \%$ & 17,57 & 8,69 \\
\hline$\Delta$ Presión & $-1,46$ & 2,19 & 7,49 \\
\hline Variación & $51,71 \%$ & $114,85 \%$ & $65,94 \%$ \\
\hline
\end{tabular}

Abbreviaturas: $\Delta$, variación de presión; DS, desviación estándar; N, newton.

Nota: Los porcentajes expresan la porción de tiempo de cargas cíclicas en la que se realiza la medición ( $25 \%=350$ ciclos; $50 \%=700$ ciclos; $75 \%=1.050$ ciclos; Final $=1.400$ ciclos).

nudos utilizados, pues, en la técnica cruzada, la presión a nivel de la huella depende directamente de la tensión entregada por el nudo, la cual no fue medida de forma cuantitativa; siempre fue realizada por el mismo operador, pero existe el riesgo de variabilidad interna. Clínicamente, es un desafío constante en la cirugía artroscópica de reparación de manguito rotador la tensión entregada tanto en nudos como en reparación con anclas sin nudos. El uso de tensiómetros en el intraoperatorio podría mejorar la reproducibilidad de estas técnicas.

Nuestros resultados son comparables con los de los estudios $^{5,13}$ biomecánicos de la reparación en DF, que han demostrado un aumento de la resistencia a la falla por carga,

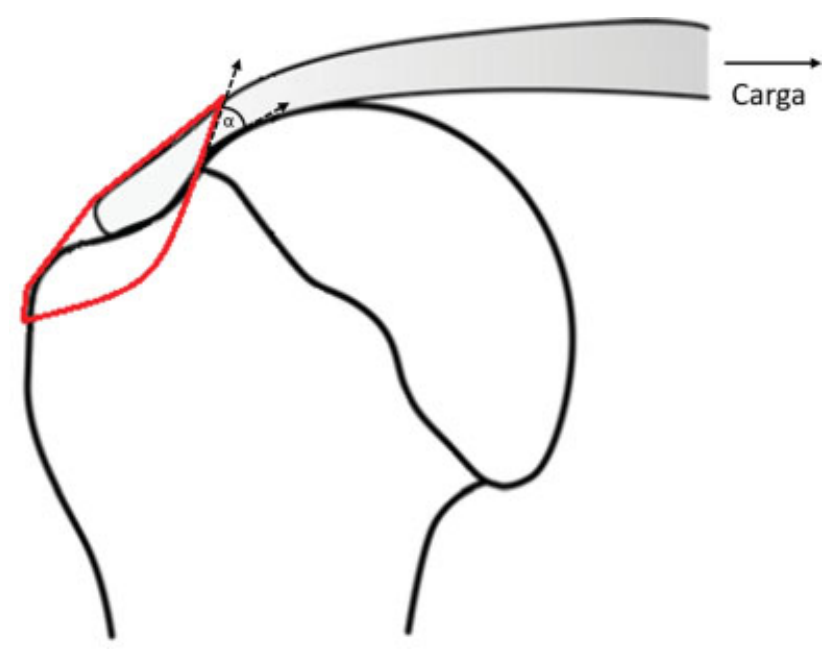

Fig.10 Esquema de autorreforzamiento en reparación transósea que ilustra un acuñamiento del ángulo entre el material de sutura y el hueso a medida que el tendón se tensiona progresivamente, y un cambio de geometría rectangular a trapezoidal de las suturas en el plano coronal a medida que aumenta la carga de tracción. áreas de contacto y presiones mejoradas, y una disminución de la formación de espacios en la interfase tendón-huella en comparación con la reparación en FU.

$\mathrm{Ng}$ et al., ${ }^{31}$ utilizando tendón de infraespinoso en un modelo porcino, compararon la distribución de presión en tres configuraciones de DF (puente de sutura; dos anclas mediales y una lateral; y un ancla medial y dos laterales), y demostraron que esta técnica no sólo da como resultado una buena área de contacto en la huella $(75 \%, 75 \%$, y $73 \%$, respectivamente), sino también que el uso de una configuración de 3 o 4 anclas produce un área de contacto similar en la huella en roturas medianas (no mayores de $1,5 \mathrm{~cm} \times 2,5 \mathrm{~cm}$ ). Estos resultados son comparables con los encontrados en nuestro estudio, y demostran al menos una equivalencia entre TOC y puente de sutura en tiempo cero respecto al área de distribución de presión en la interfase tendón-hueso. Al parecer, esta equivalencia sólo estaría dada en esta configuración, pues, en TOS, Caldow et al. ${ }^{9}$ demostraron inferioridad biomecánica de la técnica TO (área de contacto, presión de contacto, resistencia a la tracción, y la rigidez) en comparación con las configuraciones de Mason-Allen y DF.

Hinse et al. ${ }^{32}$ compararon TO con sutura, TO con cinta, y TO equivalente (TOE), y encontraron que la carga a la falla no presentaba diferencias entre TO con cinta y TO equivalente, pero que TO con sutura tenía menor resistencia estadísticamente significativa en comparación con TO equivalente, por lo que el material utilizado podría ser un factor importante para considerar. Además, a pesar de no encontrar diferencias significativas, existe una tendencia en este estudio a una mayor pérdida de cobertura en la huella con las técnicas TOs puras.

Park et al. ${ }^{12}$ compararon TO simple, FU con puntos, y FU con puntos colchoneros, y demostraron que la técnica de 
reparación del manguito rotador del túnel TO crea un contacto significativamente mayor y una mayor distribución general de la presión sobre la huella definida en comparación con las otras técnicas. Sin embargo, no compararon TOC ni TOE, que serían las técnicas más relevantes en la actualidad. Tuoheti et al. ${ }^{33}$ compararon TOS con las reparaciones en FU y en DF, y encontraron superioridad de la DF en comparación con la TOS; sin embargo, se trataba de una técnica TOS, y de la DF con puntos colchoneros, que presentan debilidades similares a las de estudio de Park et al. ${ }^{12}$

Sin embargo, estos estudio sólo evalúan propiedades biomecánicas en relación a magnitud y distribución de la presión, además de carga a la falla y, al parecer, la técnica TO tendría beneficios para la cicatrización en términos del aporte de células mesenquimales y una mejor vascularización del tendón. ${ }^{24-26}$ Urita et al. ${ }^{34}$ demostraron que la vascularización en pacientes estudiados con ultrasonido es superior en aquellos con reparación artroscópica TO en comparación con TOE.

Respecto a las limitaciones, este estudio evaluó sólo los aspectos biomecánicos en un modelo animal, por lo que los hallazgos podrían ser diferentes en un humano y en condiciones biológicas (considerar células mesenquimáticas y la irrigación). El uso de hombros cadavéricos humanos sería mejor para representar las características biomecánicas evaluadas. Por otro lado, el uso de este modelo estandariza nuestros resultados, porque cada muestra tiene seis meses de edad $\mathrm{y}$, por lo tanto, es fácilmente comparable a las demás. Esto también es cierto para la densidad mineral ósea, que no se calculó para nuestras muestras, pero habrían sido muy similares teniendo la misma edad.

Otro aspecto importante para considerar es la relevancia clínica de estos resultados, pues, a pesar de encontrar diferencias estadísticamente significativas en factores biomecánicos, la cicatrización del manguito rotador depende de muchos factores, por lo que se desconoce su impacto clínico.

\section{Conclusión}

La reparación TOC presenta mayor presión en la interfase tendón-hueso, menor pérdida de fuerza de contacto ante cargas cíclicas, y una mejor distribución de fuerza en la huella al comparar con la reparación TOS.

\section{Agradecimientos}

A nuestra familia, por el constante apoyo en nuestra labor de investigación.

\section{Referencias}

1 Jancuska J, Matthews J, Miller T, Kluczynski MA, Bisson LJ. A Systematic Summary of Systematic Reviews on the Topic of the Rotator Cuff. Orthop J Sports Med 2018;6(09): 2325967118797891. Doi: 10.1177/2325967118797891

2 Collin P, Colmar M, Thomazeau H, et al. Clinical and MRI Outcomes 10 Years After Repair of Massive Posterosuperior Rotator Cuff
Tears. J Bone Joint Surg Am 2018;100(21):1854-1863. Doi: 10.2106/JBJS.17.01190

3 Collin P, Thomazeau H, Walch G, et al. Clinical and structural outcome twenty years after repair of isolated supraspinatus tendon tears. J Shoulder Elbow Surg 2019;28(01):196-202. Doi: 10.1016/j.jse.2018.07.023

4 Piper CC, Hughes AJ, Ma Y, Wang H, Neviaser AS. Operative versus nonoperative treatment for the management of full-thickness rotator cuff tears: a systematic review and meta-analysis. J Shoulder Elbow Surg 2018;27(03):572-576. Doi: 10.1016/j. jse.2017.09.032

5 Rossi LA, Rodeo SA, Chahla J, Ranalletta M. Current Concepts in Rotator Cuff Repair Techniques: Biomechanical, Functional, and Structural Outcomes. Orthop J Sports Med 2019;7(09): 2325967119868674. Doi: 10.1177/2325967119868674

6 Chona DV, Lakomkin N, Lott A, et al. The timing of retears after arthroscopic rotator cuff repair. J Shoulder Elbow Surg 2017;26 (11):2054-2059. Doi: 10.1016/j.jse.2017.07.015

7 Haque A, Pal Singh H. Does structural integrity following rotator cuff repair affect functional outcomes and pain scores? A metaanalysis. Shoulder Elbow 2018;10(03):163-169. Doi: 10.1177/ 1758573217731548

8 Galatz LM, Ball CM, Teefey SA, Middleton WD, Yamaguchi K. The outcome and repair integrity of completely arthroscopically repaired large and massive rotator cuff tears. J Bone Joint Surg Am 2004;86(02):219-224. Doi: 10.2106/00004623-200402000-00002

9 Caldow J, Richardson M, Balakrishnan S, Sobol T, Lee PV, Ackland DC. A cruciate suture technique for rotator cuff repair. Knee Surg Sports Traumatol Arthrosc 2015;23(02):619-626. Doi: 10.1007/ s00167-014-3474-7

10 Desmoineaux P. Failed rotator cuff repair. Orthop Traumatol Surg Res 2019;105(1S)S63-S73. Doi: 10.1016/j.otsr.2018.06.012

11 Cicak N, Klobucar H, Bicanic G, Trsek D. Arthroscopic transosseous suture anchor technique for rotator cuff repairs. Arthroscopy 2006;22(05):565.e1-565.e6. Doi: 10.1016/j.arthro.2005.07.029

12 Park MC, Cadet ER, Levine WN, Bigliani LU, Ahmad CS. Tendon-tobone pressure distributions at a repaired rotator cuff footprint using transosseous suture and suture anchor fixation techniques. Am J Sports Med 2005;33(08):1154-1159. Doi: 10.1177/ 0363546504273053

13 Hohmann E, König A, Kat CJ, Glatt V, Tetsworth K, Keough N. Singleversus double-row repair for full-thickness rotator cuff tears using suture anchors. A systematic review and meta-analysis of basic biomechanical studies. Eur J Orthop Surg Traumatol 2018;28(05): 859-868. Doi: 10.1007/s00590-017-2114-6

14 Apreleva M, Ozbaydar M, Fitzgibbons PG, Warner JJ. Rotator cuff tears: the effect of the reconstruction method on threedimensional repair site area. Arthroscopy 2002;18(05): 519-526. Doi: $10.1053 /$ jars.2002.32930

15 Ma R, Chow R, Choi L, Diduch D. Arthroscopic rotator cuff repair: suture anchor properties, modes of failure and technical considerations. Expert Rev Med Devices 2011;8(03):377-387. Doi: $10.1586 /$ erd.11.4

16 Kowalsky MS, Dellenbaugh SG, Erlichman DB, Gardner TR, Levine WN, Ahmad CS. Evaluation of suture abrasion against rotator cuff tendon and proximal humerus bone. Arthroscopy 2008;24(03): 329-334. Doi: 10.1016/j.arthro.2007.09.011

17 Ntalos D, Huber G, Sellenschloh K, et al. All-suture anchor pullout results in decreased bone damage and depends on cortical thickness. Knee Surg Sports Traumatol Arthrosc 2020; $\cdots$;. Doi: 10.1007/s00167-020-06004-6

18 Godry H, Jettkant B, Seybold D, Venjakob AJ, Bockmann B. Pullout strength and failure mode of industrially manufactured and selfmade all-suture anchors: a biomechanical analysis. J Shoulder Elbow Surg 2020;29(07):1479-1483

19 Imam MA, Abdelkafy A. Outcomes following arthroscopic transosseous equivalent suture bridge double row rotator cuff 
repair: a prospective study and short-term results. SICOT J 2016; 2:7. Doi: $10.1051 /$ sicotj/2015041

20 Flanagin BA, Garofalo R, Lo EY, et al. Midterm clinical outcomes following arthroscopic transosseous rotator cuff repair. Int J Shoulder Surg 2016;10(01):3-9. Doi: 10.4103/09736042.174511

21 Garofalo R, Castagna A, Borroni M, Krishnan SG. Arthroscopic transosseous (anchorless) rotator cuff repair. Knee Surg Sports Traumatol Arthrosc 2012;20(06):1031-1035. Doi: 10.1007/ s00167-011-1725-4

22 Benson EC, MacDermid JC, Drosdowech DS, Athwal GS. The incidence of early metallic suture anchor pullout after arthroscopic rotator cuff repair. Arthroscopy 2010;26(03): 310-315. Doi: 10.1016/j.arthro.2009.08.015

23 Chillemi C, Mantovani M. Arthroscopic trans-osseous rotator cuff repair. Muscles Ligaments Tendons J 2017;7(01):19-25. Doi: 10.11138/mltj/2017.7.1.019

24 Tauber M, Koller H, Resch H. Transosseous arthroscopic repair of partial articular-surface supraspinatus tendon tears. Knee Surg Sports Traumatol Arthrosc 2008;16(06):608-613. Doi: 10.1007| s00167-008-0532-z

25 Campbell TM, Lapner P, Dilworth FJ, et al. Tendon contains more stem cells than bone at the rotator cuff repair site. J Shoulder Elbow Surg 2019;28(09):1779-1787. Doi: 10.1016/j. jse.2019.02.008

26 Kida Y, Morihara T, Matsuda K, et al. Bone marrow-derived cells from the footprint infiltrate into the repaired rotator cuff. J Shoulder Elbow Surg 2013;22(02):197-205. Doi: 10.1016/j. jse.2012.02.007

27 Burkhart SS, Adams CR, Burkhart SS, Schoolfield JD. A biomechanical comparison of 2 techniques of footprint reconstruction for rotator cuff repair: the SwiveLock-FiberChain construct versus standard double-row repair. Arthroscopy 2009; 25(03):274-281. Doi: 10.1016/j.arthro.2008.09.024

28 Andres BM, Lam PH, Murrell GA. Tension, abduction, and surgical technique affect footprint compression after rotator cuff repair in an ovine model. J Shoulder Elbow Surg 2010;19(07):1018-1027. Doi: $10.1016 /$ j.jse.2010.04.005

29 Mahar AT, Moezzi DM, Serra-Hsu F, Pedowitz RA. Comparison and performance characteristics of 3 different knots when tied with 2 suture materials used for shoulder arthroscopy. Arthroscopy 2006;22(06):614.e1-614.e2. Doi: 10.1016/j.arthro.2006.02.005

30 Wüst DM, Meyer DC, Favre P, Gerber C. Mechanical and handling properties of braided polyblend polyethylene sutures in comparison to braided polyester and monofilament polydioxanone sutures. Arthroscopy 2006;22(11):1146-1153. Doi: 10.1016/j.arthro.2006.06.013

$31 \mathrm{Ng}$ SHA, Tan CHJ. Double-row repair of rotator cuff tears: Comparing tendon contact area between techniques. World J Orthop 2020;11(01):10-17. Doi: 10.5312/wjo.v11.i1.10

32 Hinse S, Ménard J, Rouleau DM, Canet F, Beauchamp M. Biomechanical study comparing 3 fixation methods for rotator cuff massive tear: Transosseous No. 2 suture, transosseous braided tape, and double-row. J Orthop Sci 2016;21(06): 732-738. Doi: 10.1016/j.jos.2016.07.001

33 Tuoheti Y, Itoi E, Yamamoto N, et al. Contact area, contact pressure, and pressure patterns of the tendon-bone interface after rotator cuff repair. Am J Sports Med 2005;33(12): 1869-1874. Doi: 10.1177/0363546505278256

34 Urita A, Funakoshi T, Horie T, Nishida M, Iwasaki N. Difference in vascular patterns between transosseous-equivalent and transosseous rotator cuff repair. J Shoulder Elbow Surg 2017;26 (01):149-156. Doi: 10.1016/j.jse.2016.06.010 